\title{
Resistência do arroz de terras altas ao alumínio
}

\author{
Cleber M. Guimarães ${ }^{1}$, Péricles de C. F. Neves ${ }^{1}$, Luís F. Stone ${ }^{1}$ \& Francisco J. P. Zimmermann ${ }^{2}$
}

\begin{abstract}
RESUMO
Objetivou-se, com este trabalho, identificar indicadores de resistência à toxicidade de alumínio em arroz de terras altas e linhagens com baixa susceptibilidade a esta toxicidade. Avaliaram-se 48 linhagens de arroz em solução nutritiva e dois níveis de estresse de alumínio: 0 e $40 \mathrm{mg} \mathrm{dm}^{-3}$ de Al; após 21 dias de teste, estimaram-se a massa da matéria seca das raízes e do dossel, o comprimento máximo das raízes e a altura das plantas e, também, índices de susceptibilidade à toxicidade de alumínio. Verificou-se que todos os indicadores de susceptibilidade ao alumínio, exceto aqueles relacionados à variabilidade do $\mathrm{pH}$, se correlacionaram significativamente. O índice de susceptibilidade do crescimento radicular $\left(\mathrm{S}_{\mathrm{Rcm}}\right)$ por se considerar, no seu cálculo, o crescimento radicular com e sem estresse de Al e a pressão de estresse de Al em que as linhagens foram avaliadas, constitui-se em importante parâmetro a ser usado na seleção para resistência à toxicidade ao alumínio. Conforme a distribuição das linhagens em quartis, delimitados pelo comprimento radicular superior a $47 \mathrm{~cm}$ e pelo $S_{\text {Rcm }}$ menor que 0,92, selecionou-se o grupo de linhagens, CNA4120, CNA4164 e CNA1383, que apresenta raízes bem desenvolvidas, tanto na ausência como na presença de alumínio.
\end{abstract}

Palavras-chave: Oryza sativa, índice de susceptibilidade, raízes, linhagens sensíveis e não sensíveis ao Al

\section{Aluminum resistance of upland rice}

\begin{abstract}
The objective of this work was to identify indicators of resistance of aluminum toxicity in upland rice as well as lines with low susceptibility to this toxicity. Fifty one rice lines were evaluated in greenhouse, in nutrient solution, under two levels: 0 and $40 \mathrm{mg} \mathrm{dm}^{-3}$ of Al. Data of dry weight of roots and aerial parts, length of roots and plant height were obtained 21 days later. Also, the index of susceptibility to aluminum toxicity was calculated. The results showed significant correlation between all indicators with each other, except to those related to $\mathrm{pH}$ variability. Since root length susceptibility index $\left(\mathrm{S}_{\mathrm{Rcm}}\right)$ was calculated taking into consideration the root growth with and without stress and the pressure of aluminum stress under which the genotypes were evaluated, it can be considered a reliable tool for selection of cultivars/lines for resistance to aluminum toxicity. According to the distribution of lines in quarters delimited by root length higher than $47 \mathrm{~cm}$ and by $S_{\text {Rcm }}$ lower than 0.92, the lines CNA4120, CNA4164, and CNA1383 were selected, which have roots well developed in the absence or presence of Al.
\end{abstract}

Key words: Oryza sativa, susceptibly index, roots, Al-sensitive and resistant lines

1 Embrapa Arroz e Feijão, CP 179, CEP 75375-000, Santo Antônio de Goiás, GO. Fone: (62) 533-2178. E-mail: cleber@cnpaf.embrapa.br ${ }^{2}$ Universidad de La Sabana, Facultad de Ingeniería, Km 21 Autopista Norte de Bogotá, D.C., Chia, Cundinamarca, Colombia. Fone: 861-5555 / 861-6666. Email: francisco.dfeilsticker@unisabana.edu.co 


\section{INTRODUÇÃO}

A toxicidade de alumínio é um significativo fator limitante ao crescimento das plantas em solos ácidos, cuja produtividade é aumentada com a redução da sua atividade (Fageria et al., 1988). Devido à toxicidade de alumínio, a inibição do crescimento radicular é o primeiro sintoma facilmente observado (Foy, 1988; Kochian, 1995), a qual ocorre dentro de uma a duas horas após a exposição à concentração tóxica do alumínio (Clarkson, 1965; Kochian, 1995). O alumínio interfere na divisão celular que ocorre nos pontos de crescimento das raízes, torna a parede celular rígida pela deposição de pectina, reduz a duplicação do DNA, fixa o fósforo na superfície radicular, inibe a respiração radicular e interfere na atividade enzimática responsável pela fosforilação do açúcar e deposição de polissacarídeos na parede celular (Foy, 1992). O primeiro sítio de fixação de alumínio se situa na coifa e na mucilagem radicular (Bennet et al. 1985). Por outro lado, estudos de tolerância ao alumínio têm estabelecido que as plantas podem resistir aos seus efeitos tóxico; esta resistência se dá pela tolerância interna e pelo escape externo (Foy, 1988; Taylor, 1991). A quelatação do alumínio pelos ácidos orgânicos excretados pelas raízes em resposta à toxicidade de alumínio é um mecanismo bastante evidente de escape (Delhaize et al., 1993; Pellet et al., 1995). A adsorsão do alumínio pelas cargas negativas da mucilagem radicular, que previne a penetração do alumínio no meristema radicular, é outro exemplo de escape à toxicidade do alumínio (Horst et al., 1982; Li et al., 2000). Tem-se observado também que a exudação de mucilagens com propriedades químicas para se ligar ao alumínio, pelas células periféricas do sistema radicular, protege os pontos de crescimento da planta quanto à ação tóxica do alumínio (Miyasake \& Hawes, 2001). Entre os vários mecanismos de tolerância ao alumínio cita-se a capacidade das plantas manterem, em suas raízes ou na parte aérea, níveis adequados de certos macronutrientes e micronutrientes. As cultivares tolerantes de arroz apresentam, quase sempre, na presença de níveis tóxicos de alumínio, teores de fósforo e cálcio mais elevados que as cultivares sensíveis (Fageria, 1985; Sivaguru \& Paliwal, 1993; Mendonça et al., 2003).

Uma alternativa para contornar o problema é a neutralização, pelo menos em parte, do alumínio trocável da solução do solo pela aplicação de calcário mas, em áreas distantes das suas fontes, os gastos de correção se tornam excessivamente elevados; adicionalmente, há dificuldades para a sua aplicação a profundidades maiores que $30 \mathrm{~cm}$. Em tais condições, é desejável a adoção de cultivares mais tolerantes à toxicidade do elemento (Fageria, 1982).

Com este trabalho, objetivou-se identificar indicadores de resistência à toxicidade de alumínio e linhagens portadoras de resistência à toxicidade deste elemento, mas que apresentem bom desenvolvimento do sistema radicular, na sua ausência.

\section{MATERIAL E MÉTODOS}

Foram avaliadas 48 linhagens de arroz (Oryza sativa L.) provenientes do Banco Ativo de Germoplasma da Embrapa
Arroz e Feijão, as quais foram colocadas para germinar em bandejas de plástico com areia esterilizada. Após oito dias, as plântulas, selecionadas quanto à uniformidade de sistema radicular e dossel, foram transplantadas para vasos de polietileno pretos, contendo oito litros de solução nutritiva aerada, que eram equipados com placas de acrílico que, por sua vez, suportavam quatro discos, distribuídos equidistantemente, com quatro perfurações cada um, nas quais foram acomodadas, individualmente, as plântulas, com o auxílio de algodão. Usou-se a solução nutritiva de Furlani \& Furlani (1988) para arroz, modificada para conter as seguintes concentrações de $\mathrm{Al}, 0$ e $40 \mathrm{mg} \mathrm{dm}^{-3}$ de Al, sob a forma de $\mathrm{AlCl}_{3} \cdot 6 \mathrm{H}_{2} 0$ (Fageria \& Zimmermann, 1979). Os valores de $\mathrm{pH}$ das soluções nutritivas, com 0 e $40 \mathrm{mg} \mathrm{dm}^{-3}$ de $\mathrm{Al}$ foram, inicialmente, padronizados em 4,0, pela adição de $\mathrm{HCl} 0,1 \mathrm{~N}$ ou $\mathrm{NaOH} 0,1 \mathrm{~N}$, e não mais corrigidos durante a condução do experimento. Após 21 dias do transplante as plantas foram colhidas e determinadas as massas da matéria seca das raízes e do dossel, após serem secadas em estufa a $70{ }^{\circ} \mathrm{C}$, até massa constante. Determinou-se também o comprimento máximo das raízes e a altura das plantas e se adotou o índice de susceptibilidade $\mathrm{S}$, de Fisher \& Maurer (1978), adaptado para estresse de alumínio, conforme as equações seguintes:

$$
\begin{gathered}
\mathrm{S}=\left(\mathrm{Y}_{\mathrm{s} / \mathrm{Al}}-\mathrm{Y}_{\mathrm{c} / \mathrm{Al}}\right) /\left(\mathrm{Y}_{\mathrm{s} / \mathrm{Al}} * \mathrm{D}\right) \\
\mathrm{D}=1-\left(\mathrm{X}_{\mathrm{c} / \mathrm{Al}} / \mathrm{X}_{\mathrm{s} / \mathrm{Al}}\right)
\end{gathered}
$$

em que:

D - severidade do estresse aplicado

$\mathrm{Y}_{\mathrm{s} / \mathrm{Al}}$ e $\mathrm{Y}_{\mathrm{c} / \mathrm{Al}}$ - matérias secas e os comprimentos individuai das raízes ou do dossel das linhagens na ausência e presença de alumínio, respectivamente

$\mathrm{X}_{\mathrm{s} / \mathrm{Al}}$ e $\mathrm{X}_{\mathrm{c} / \mathrm{Al}}$ - matérias secas e os comprimentos médios das linhagens nos ambientes considerados.

O indicador é tanto menos afetado pelo nível de estresse induzido pela toxicidade do alumínio quanto menor o $\mathrm{S}$ avaliado para aquele indicador. Calculou-se o índice de susceptibilidade do crescimento radicular $\left(\mathrm{S}_{\mathrm{Rcm}}\right)$ e do dossel $\left(\mathrm{S}_{\mathrm{Dcm}}\right)$, e do acúmulo de massa seca das raízes $\left(\mathrm{S}_{\mathrm{Rg}}\right)$ e do dossel $\left(\mathrm{S}_{\mathrm{Dg}}\right)$. $\mathrm{O}$ índice também foi usado para avaliar a capacidade das raízes em alterar o pH da solução nutritiva, avaliado ao final do período de teste.

Para finalidade de seleção, as linhagens foram distribuídas em quartis, delimitados pela média do comprimento das raízes (Raízes $\mathrm{cm}_{\mathrm{cm}}$ ), sem o estresse de toxicidade de alumínio, acrescida de $75 \%$ do seu desvio padrão, e a média do seu índice de susceptibilidade à toxicidade de alumínio $\left(\mathrm{S}_{\mathrm{Rcm}}\right)$ diminuído de $25 \%$ do seu desvio padrão.

\section{RESULTADOS E DISCUSSÃO}

Verificou-se grande variabilidade entre as linhagens de arroz de terras altas, quanto à resistência à toxicidade ao alumínio (Figura 1). Resultados semelhantes foram observados por Fageria \& Zimmermann (1979) e Fageria (1985) para a 
CNA 5167

$40 \mathrm{mg} \mathrm{dm}^{-3} \mathrm{Al}$

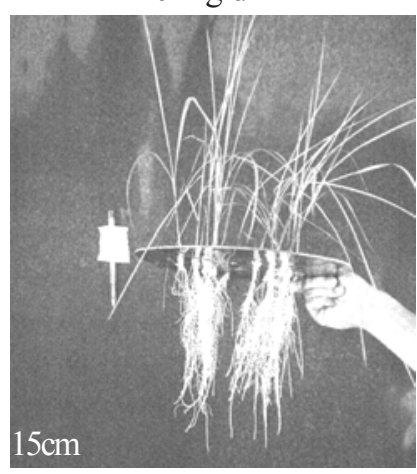

CNA 5167

$0 \mathrm{mg} \mathrm{dm}^{-3} \mathrm{Al}$

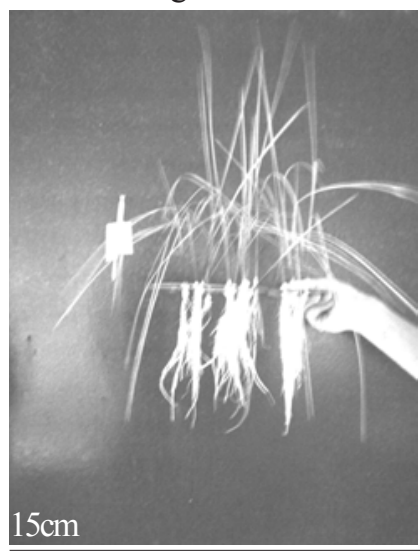

CNA 2865

$40 \mathrm{mg} \mathrm{dm}^{-3} \mathrm{Al}$

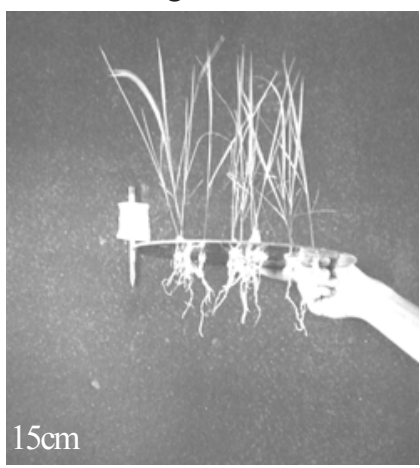

CNA 2865

$0 \mathrm{mg} \mathrm{dm}^{-3} \mathrm{Al}$

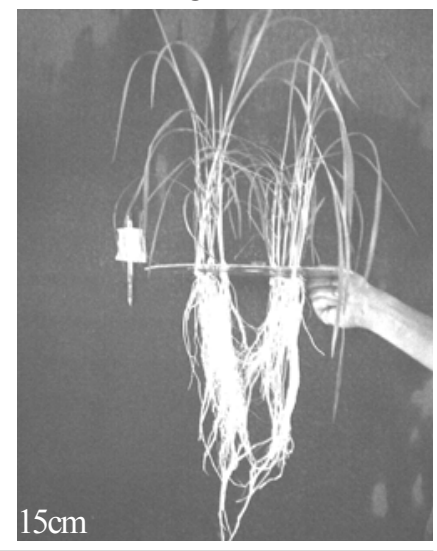

Figura 1. Linhagens com diferentes susceptibilidades à toxicidade de alumínio

cultura do arroz. Esta resistência, medida pela massa da matéria seca das raízes (Raízes $\mathrm{g}$ ) e do dossel (Plantas $\mathrm{g}$ ), comprimento das raízes (Raízes $\left.{ }_{\mathrm{cm}}\right)$ e do dossel (Plantas $\mathrm{cm}_{\mathrm{cm}}$ ) das linhagens de arroz de terras altas, em $40 \mathrm{mg} \mathrm{dm}^{-3}$ de alumínio, e índices de susceptibilidade ao alumínio e de alterações do $\mathrm{pH}$ da solução nutritiva, das linhagens mais contrastantes, são apresentados na Tabela 1. Notou-se que todos os indicadores usados, exceto o $\mathrm{pH}$, se correlacionaram significativamente (Tabela 2), sugerindo que esta variável não foi eficiente em discriminar as linhagens tolerantes das sensíveis ao alumínio.

Por outro lado, Foy et al. (1965) e Mendonça et al. (2005) observaram que as cultivares de trigo e arroz, respectivamente, tolerantes ao alumínio, são capazes de aumentar o $\mathrm{pH}$ da solução nutritiva, comparativamente com as sensíveis, quando avaliadas na presença deste elemento.

No caso do arroz, o crescimento e o acúmulo de matéria seca, tanto do sistema radicular como do dossel, assim como os seus índices de susceptibilidade, foram mais eficientes na discriminação das linhagens podendo ser usados na seleção daquelas com maior resistência ao alumínio; entretanto, considerando-se que o alumínio atua diretamente sobre o sistema radicular, no seu crescimento, através da ação sobre a divisão celular (Rout et al., 2001) sugere-se priorizar variáveis que quantifiquem o comportamento radicular das linhagens quando submetidas ao estresse de toxicidade de alumínio. Entre os indicadores de susceptibilidade radicular à toxicida- de de alumínio, o Raízes ${ }_{\mathrm{cm}}$, em condições de estresse de alumínio, e o $\mathrm{S}_{\mathrm{Rcm}}$, apresentaram o mais alto coeficiente de correlação, -0,8282. Considerando-se que no cálculo do $\mathrm{S}_{\mathrm{Rcm}}$ são computados o crescimento radicular com e sem o estresse de alumínio e a pressão de estresse em que as linhagens foram avaliadas, este indicador se constitui em importante parâmetro na seleção daquelas linhagens com maior resistência à toxicidade ao alumínio.

Considerou-se, na seleção das linhagens, o comprimento radicular sem o estresse de alumínio, $0 \mathrm{mg} \mathrm{dm}^{-3}$ do elemento, pois é desejável que apresentem resistência à toxicidade de alumínio mas também sistema radicular bem desenvolvido na ausência de concentrações tóxicas do elemento. Conforme a distribuição das linhagens em quartis, selecionaram-se dois grupos: o primeiro se compunha das linhagens CNA4120 (CNA092-BM10-BM27p-3), CNA4164 (CNA511-2-B-2) e CNA1383 (IPEACO 162) caracterizados por apresentar Raízes ${ }_{\mathrm{cm}}$ acima de $47 \mathrm{~cm}$ (média do comprimento das raízes, sem o estresse de toxicidade de alumínio, acrescida de $75 \%$ do seu desvio padrão) e $\mathrm{S}_{\mathrm{Rcm}}$ inferior a 0,92 (média do seu índice de susceptibilidade à toxicidade de alumínio, diminuído de $25 \%$ do seu desvio padrão), ou seja, apresentam sistema bem desenvolvido, tanto na ausência como na presença de alumínio; o outro grupo, pelas linhagens CNA6430 (Dourado Precoce), CNA4108 (CNA108-B-28-8-2-B-2), CNA4166 (CNA095-BM30-BM9-25), CNA4181 (CNA425-BM46-1-B-1), CNA5164 (CNA095-BM30-BM27P-15-2), CNA5165 (CNA095BM30-BM27P-17-1), CNA4105 (CNA511-16-B-6), CNA1168 (Fernandes), CNA4125 (CNA092-BM11-BM19p-4) e CNA4150 (CNA515-3-1), que apresentam Raízes ${ }_{\mathrm{cm}}$ acima de $47 \mathrm{~cm}$, porém $\mathrm{S}_{\mathrm{Rcm}}$ superior a 0,92 , ou seja, sistema bem desenvolvido na ausência de toxicidade de alumínio; entretanto, não suportam a presença tóxica do mesmo (Figura 2). Os outros quartis, por enquadrarem as linhagens que apresentam comprimento radicular ineficientes na ausência de toxicidade de alumínio, não foram considerados.

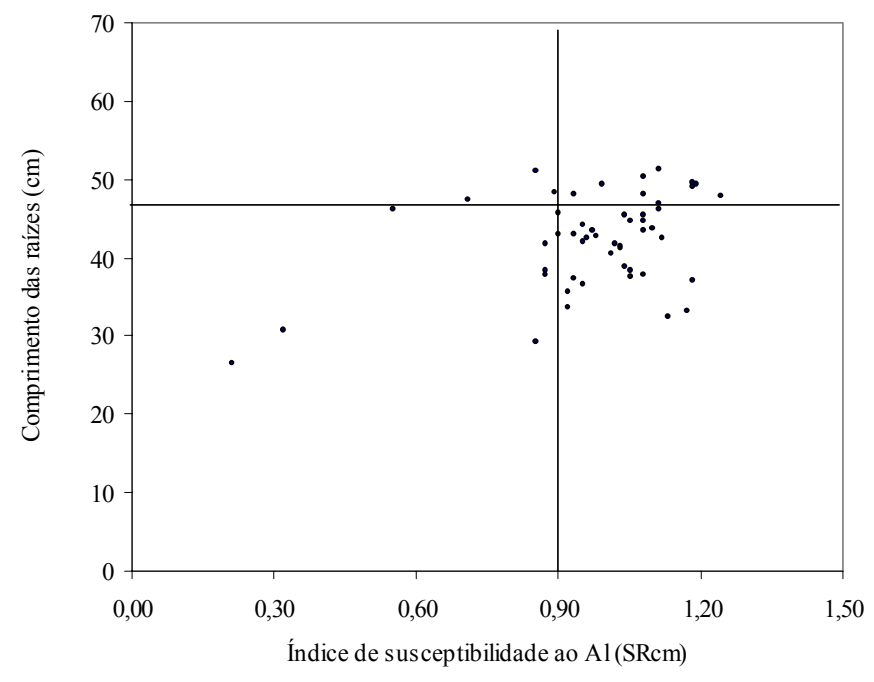

Figura 2. Distribuição das linhagens em quartis delimitados pelo comprimento radicular (Raízes ${ }_{\mathrm{cm}}$ ) em $0 \mathrm{mg} \mathrm{dm}^{-3}$ de alumínio e pelo índice de susceptibilidade do crescimento radicular à toxicidade do alumínio $\left(S_{\mathrm{Rcm}}\right)$, nos pontos determinados pela média de Raízes ${ }_{\mathrm{cm}}+75 \%$ do seu desvio padrão e pela média de $\mathrm{S}_{\mathrm{Rcm}}-25 \%$ do seu desvio padrão 
Tabela 1. Massa da matéria seca das raízes (Raízes $\mathrm{g}$ ) e do dossel (Plantas $)$, comprimento das raízes (Raízes ${ }_{\mathrm{cm}}$ ) e do dossel (Plantas ${ }_{\mathrm{cm}}$ ) das linhagens de arroz de terras altas, em $40 \mathrm{mg} \mathrm{dm}^{-3}$ de alumínio e índices de susceptibilidade ao alumínio* e de alterações do $\mathrm{pH}$ da solução nutritiva ${ }^{1}$

\begin{tabular}{|c|c|c|c|c|c|c|c|c|c|c|c|c|c|c|c|c|c|c|}
\hline $\begin{array}{l}\mathrm{N}^{0} \text { de } \\
\text { controle }\end{array}$ & Nome Comum & $\begin{array}{l}\text { Raízes }_{g} \\
\text { (g) }\end{array}$ & & $\begin{array}{l}\text { Plantas } \\
\text { (g) }\end{array}$ & & $\begin{array}{l}\text { Raízes }_{\mathrm{cn}} \\
\text { (cm) }\end{array}$ & & $\begin{array}{l}\text { Plantas }_{\text {rn }} \\
\text { (cm) }\end{array}$ & & $\mathrm{S}_{\mathrm{Rcm}}$ & & $S_{D c m}$ & & $\mathrm{~S}_{\mathrm{Rg}}$ & & $\mathrm{S}_{\mathrm{Dg}}$ & & $\mathrm{S}_{\mathrm{pH}}$ \\
\hline CNA 3178 & ITA 235 & $0,098 \mathrm{C}$ & & 0,401 & A & 22,38 & B & 41,38 & A & $0,21 \mathrm{D}$ & D & 0,40 & & $-2,26$ & C & $-2,56$ & D & $-1,25 \mathrm{M}$ \\
\hline CNA 4140 & CNA 092-BM11-BM19p-1-Rio Paraguaio & $0,178 \mathrm{~B}$ & B & 0,256 & C & 23,38 & B & 42,13 & A & $0,32 \mathrm{D}$ & D & 0,41 & $\mathrm{D}$ & $-3,94$ & $\mathrm{D}$ & $-3,19$ & D & $-0,08 \quad \mid$ \\
\hline CNA 4411 & N.7367 [CA 435 X IAC 25] & $0,088 \mathrm{C}$ & C & 0,271 & C & 30,13 & A & 41,13 & A & $0,55 \mathrm{c}$ & C & 0,31 & D & 1,07 & B & 1,14 & B & $0,91 \mathrm{G}$ \\
\hline CNA 4120 & CNA 092-BM10-BM27p-3 & $0,155 \mathrm{~B}$ & B & 0,254 & C & 26,5 & A & 39,88 & A & 0,710 & C & 0,61 & C & 1,23 & B & 1,69 & B & $0,08 \quad$ I \\
\hline CNA 1383 & IPEAC0 162 & $0,085 \mathrm{C}$ & C & 0,094 & $\mathrm{D}$ & 24,13 & B & 45,25 & A & $0,85 \mathrm{~B}$ & B & 0,22 & D & 2,32 & A & 3,33 & A & $1,69 \mathrm{E}$ \\
\hline CNA 6571 & IPEAC0 562 (ECAD) & $0,210 \mathrm{~B}$ & B & 0,449 & A & 13,13 & D & 34,13 & B & $0,85 \mathrm{~B}$ & B & 1,10 & B & $-3,97$ & $\mathrm{D}$ & $-2,27$ & D & $-0,73 \mathrm{~L}$ \\
\hline CNA 5021 & CNA 1211-BM-B-3-B & $0,170 \mathrm{~B}$ & B & 0,316 & B & 20,00 & B & 28,38 & C & $0,87 \mathrm{~B}$ & B & 1,29 & B & 0,52 & B & 1,04 & B & $-1,66 \mathrm{~N}$ \\
\hline CNA 4476 & N.7454[RS 25X1AC 25] & $0,128 \mathrm{C}$ & C & 0,239 & C & 18,25 & C & 30,88 & C & $0,87 \mathrm{~B}$ & B & 1,15 & B & 0,64 & B & 1,06 & B & $1,45 \mathrm{~F}$ \\
\hline CNA 4179 & CNA 511-16-B-3 & 0,198 B & B & 0,279 & C & 18,13 & C & 34,38 & B & $0,87 \mathrm{~B}$ & B & 0,99 & B & $-0,25$ & B & 0,76 & B & $4,35 \mathrm{~B}$ \\
\hline CNA 4164 & CNA 511-2-B-2 & $0,175 \mathrm{~B}$ & B & 0,291 & C & 22,38 & B & 31,00 & C & $0,89 \mathrm{~B}$ & B & 1,09 & B & 0,37 & B & 0,67 & B & $0,02 \quad$ \\
\hline CNA 5167 & CNA 095-BM30-BM27P-80-2 & $0,273 \mathrm{~A}$ & A & 0,459 & A & 20,88 & B & 38,88 & A & $0,90 \mathrm{~B}$ & B & 0,74 & C & $-1,35$ & C & $-0,34$ & C & $1,41 \mathrm{~F}$ \\
\hline CNA 5166 & CNA 095-BM30-BM27P-42-1/DOURADÃO & $0,208 \mathrm{~B}$ & B & 0,444 & A & 19,50 & B & 35,63 & B & $0,90 \mathrm{~B}$ & B & 0,92 & C & $-0,2$ & B & $-0,51$ & C & $1,41 \mathrm{~F}$ \\
\hline CNA 2739 & Paulistinha & $0,145 \mathrm{~B}$ & B & 0,201 & C & 16,13 & C & 35,00 & B & $0,92 \mathrm{~B}$ & B & 1,15 & B & 0,6 & B & 1,72 & B & $-1,54 \mathrm{~N}$ \\
\hline CNA 4640 & TOX 1785-19-18 & $0,090 \mathrm{C}$ & C & 0,211 & C & 13,88 & $\mathrm{D}$ & 36,25 & B & $0,92 \mathrm{~B}$ & B & 0,85 & C & 0,66 & B & 2,11 & A & $0,23 \mathrm{H}$ \\
\hline CNA 4166 & CNA 095-BM30-BM9-25 & $0,195 \mathrm{~B}$ & B & 0,374 & B & 20,88 & B & 35,13 & B & $0,93 \mathrm{~B}$ & B & 1,06 & B & 0,38 & B & 0,48 & B & $5,74 \mathrm{~A}$ \\
\hline CNA 5163 & CNA 82 M0 75 & $0,333 \mathrm{~A}$ & A & 0,456 & A & 17,88 & C & 35,75 & B & $0,93 \mathrm{~B}$ & B & 0,93 & C & $-4,09$ & D & $-1,18$ & C & $0,5 \mathrm{H}$ \\
\hline CNA 2523 & MATÃO & $0,235 \mathrm{~B}$ & B & 0,334 & B & 19,38 & B & 30,00 & C & $0,95 \mathrm{~B}$ & B & 1,09 & B & 0,15 & B & 0,74 & B & $2,88 \mathrm{C}$ \\
\hline CNA 4634 & TOX 1369-18-1 & $0,158 \mathrm{~B}$ & B & 0,359 & B & 17,88 & C & 34,25 & B & $0,95 \mathrm{~B}$ & B & 0,94 & C & 0,63 & B & 0,02 & C & $4,31 \quad B$ \\
\hline CNA 2826 & PONTA PRETA & 0,188 B & B & 0,449 & A & 15,00 & C & 33,75 & B & $0,95 \mathrm{~B}$ & B & 1,19 & B & 0,12 & B & 0,69 & B & $2,63 \mathrm{D}$ \\
\hline CNA 0486 & BATATAIS & $0,175 \mathrm{~B}$ & B & 0,339 & B & 17,88 & C & 31,00 & C & $0,96 \mathrm{~B}$ & B & 1,06 & B & 0,07 & B & 0,2 & C & $4,31 \quad B$ \\
\hline CNA 2836 & PÉROLA & $0,240 \mathrm{~B}$ & B & 0,381 & B & 17,00 & C & 37,75 & B & $0,97 \mathrm{~B}$ & B & 0,87 & C & $-0,95$ & C & 0,53 & B & $-0,09 \quad$ । \\
\hline CNA 799 & Catalão & $0,150 \mathrm{~B}$ & B & 0,341 & B & 17,38 & C & 33,63 & B & $0,98 \mathrm{~B}$ & B & 0,91 & C & 0,71 & B & 0,72 & B & $5,67 \mathrm{~A}$ \\
\hline CNA 4105 & CNA 511-16-B-6 & $0,070 \mathrm{C}$ & C & 0,204 & C & 19,63 & B & 28,63 & C & $0,99 \mathrm{~B}$ & B & 1,35 & B & 1,5 & B & 1,53 & B & $-0,07 \quad$ । \\
\hline CNA 3289 & IREM 247 & $0,098 \mathrm{C}$ & C & 0,216 & C & 15,88 & C & 29,88 & C & $1,01 \mathrm{~B}$ & B & 0,86 & C & 1,12 & B & 1,08 & B & $1,41 \mathrm{~F}$ \\
\hline CA 780284 & JAVANÊS & $0,053 \mathrm{C}$ & C & 0,191 & C & 15,13 & C & 31,25 & C & $1,02 \mathrm{~A}$ & A & 1,13 & B & 2,41 & A & 2,21 & A & $0,36 \mathrm{H}$ \\
\hline CNA 4617 & TOX 1022-4-2 & $0,178 \mathrm{~B}$ & B & 0,396 & A & 15,63 & C & 17,25 & $\mathrm{E}$ & $1,03 \mathrm{~A}$ & A & 1,98 & A & 0,17 & B & $-0,26$ & C & $1,45 \mathrm{~F}$ \\
\hline CNA 5171 & CNA 511-8-B-4 & $0,080 \mathrm{C}$ & C & 0,229 & C & 14,63 & C & 26,50 & C & $1,03 \mathrm{~A}$ & A & 1,36 & B & 1,58 & B & 1,54 & B & $-0,09 \quad$ । \\
\hline CNA 4180 & CNA 511-6-B-3 & $0,173 \mathrm{~B}$ & B & 0,429 & A & 16,25 & C & 34,00 & B & $1,04 \mathrm{~A}$ & A & 1,15 & B & $-1,97$ & C & $-0,52$ & C & $0,26 \mathrm{H}$ \\
\hline CNA 4144 & CNA 449-BM15-1-B-4 & $0,115 \mathrm{C}$ & C & 0,221 & C & 13,63 & D & 37,88 & B & $1,04 \mathrm{~A}$ & A & 0,77 & C & $-1,03$ & C & 0,19 & C & $2,61 \mathrm{D}$ \\
\hline CNA 2721 & PRATÃO PRECOCE & $0,078 \mathrm{C}$ & C & 0,289 & C & 14,25 & $\mathrm{D}$ & 41,00 & A & $1,05 \mathrm{~A}$ & A & 0,64 & C & 2,60 & A & 1,95 & A & $-3,01 P$ \\
\hline CNA 4171 & CNA 511-16-B-5 & $0,155 \mathrm{~B}$ & B & 0,326 & B & 13,63 & D & 29,25 & C & $1,05 \mathrm{~A}$ & A & 1,35 & B & 0,25 & B & 0,83 & B & $2,88 \mathrm{C}$ \\
\hline CNA 1462 & IRAT 13 & $0,033 \mathrm{D}$ & D & 0,176 & C & 13,13 & D & 25,13 & D & $1,05 \mathrm{~A}$ & A & 1,29 & B & 2,94 & A & 2,57 & A & $\begin{array}{ll}-1,91 & 0\end{array}$ \\
\hline CNA 4125 & CNA 092-BM11-BM19p-4 & $0,080 \mathrm{C}$ & C & 0,286 & C & 17,25 & C & 34,88 & B & $1,08 \mathrm{~A}$ & A & 0,97 & B & 1,24 & B & 0,29 & C & $1,41 \mathrm{~F}$ \\
\hline CNA 2720 & PRATÃO PRECOCE & $0,118 \mathrm{C}$ & C & 0,296 & C & 16,63 & C & 31,38 & C & $1,08 \mathrm{~A}$ & A & 1,14 & B & 0,65 & B & 0,13 & C & $2,88 \mathrm{C}$ \\
\hline CNA 4181 & CNA 425-BM46-1-B-1 & $0,005 \mathrm{D}$ & D & 0,114 & D & 16,25 & C & 36,75 & B & $1,08 \mathrm{~A}$ & A & 0,94 & C & 3,08 & A & 2,96 & A & $0,06 \quad$ I \\
\hline CNA 4178 & CNA 444-B38-7-B-4 & $0,163 \mathrm{~B}$ & B & 0,301 & B & 15,63 & C & 29,63 & C & $1,08 \mathrm{~A}$ & A & 1,08 & B & 0,65 & B & 0,66 & B & $2,88 \mathrm{C}$ \\
\hline CNA 6564 & IAC 164 (ECAD) & $0,213 \mathrm{~B}$ & B & 0,391 & B & 15,00 & C & 34,13 & B & $1,08 \mathrm{~A}$ & A & 1,04 & B & $-0,28$ & B & 0,41 & B & $-0,15 \quad$ । \\
\hline CNA 2042 & IAC 0047 & $0,183 \mathrm{~B}$ & B & 0,334 & B & 14,63 & C & 32,63 & B & $1,10 \mathrm{~A}$ & A & 0,72 & C & $-0,30$ & B & $-0,38$ & C & $2,88 \mathrm{C}$ \\
\hline CNA 4150 & CNA 515-3-1 & $0,125 \mathrm{C}$ & C & 0,281 & C & 16,63 & C & 34,25 & B & $1,11 \mathrm{~A}$ & A & 1,14 & B & 0,80 & B & 0,86 & B & $2,88 \mathrm{C}$ \\
\hline CNA 6430 & DOURADO PRECOCE & $0,188 \mathrm{~B}$ & B & 0,341 & B & 15,38 & C & 35,00 & B & $1,11 \mathrm{~A}$ & A & 0,87 & C & 0,50 & B & 0,66 & B & $-0,07 \quad$ । \\
\hline CNA 4148 & CNA 104-B-68-B-2 & $0,078 \mathrm{C}$ & C & 0,506 & A & 14,50 & C & 38,75 & A & $1,11 \mathrm{~A}$ & A & 0,77 & C & 1,98 & A & $-0,39$ & C & $-0,80 M$ \\
\hline CNA 3373 & IRAT 11 & $0,073 \mathrm{C}$ & C & 0,209 & C & 13,50 & $\mathrm{D}$ & 23,50 & $\mathrm{D}$ & $1,12 \mathrm{~A}$ & A & 1,16 & B & 1,40 & B & 1,06 & B & $5,74 \mathrm{~A}$ \\
\hline CNA 8521 & CNA 104-B-18-PY-1-2 & $0,063 \mathrm{C}$ & C & 0,206 & C & 8,50 & D & 41,50 & A & $1,17 \mathrm{~A}$ & A & 0,85 & C & 2,66 & A & 2,41 & A & $-1,38 M$ \\
\hline CNA 5164 & CNA 095-BM30-BM27P-15-2 & $0,100 \mathrm{C}$ & C & 0,259 & C & 13,88 & D & 34,38 & B & $1,18 \mathrm{~A}$ & A & 1,03 & B & 1,26 & B & 1,47 & B & $2,88 \mathrm{C}$ \\
\hline CNA 1168 & FERNANDES & $0,160 \mathrm{~B}$ & B & 0,319 & B & 13,50 & D & 30,25 & C & $1,18 \mathrm{~A}$ & A & 1,18 & B & 0,62 & B & 0,96 & B & $-0,07 \quad$ । \\
\hline CNA 2865 & QUATRO MESES & $0,183 \mathrm{~B}$ & B & 0,276 & C & 10,63 & $\mathrm{D}$ & 26,50 & C & $1,18 \mathrm{~A}$ & A & 1,43 & B & 0,34 & B & 1,34 & B & $-1,54 \mathrm{~N}$ \\
\hline CNA 5165 & CNA 095-BM30-BM27P-17-1 & $0,228 B$ & B & 0,351 & B & 13,25 & D & 34,13 & B & $1,19 \mathrm{~A}$ & A & 0,96 & B & $-2,17$ & C & $-1,36$ & C & $-0,46 \mathrm{~J}$ \\
\hline CNA 4108 & CNA 108-B-28-8-2-B-2 & $0,028 \mathrm{D}$ & D & 0,344 & B & 11,13 & D & 34,75 & B & $1,24 \mathrm{~A}$ & A & 1,07 & B & 3,18 & A & 1,48 & $B$ & $1,44 \mathrm{~F}$ \\
\hline
\end{tabular}

${ }^{*} \mathrm{~S}_{\mathrm{Rcm}}, \mathrm{S}_{\mathrm{Dcm}}, \mathrm{S}_{\mathrm{Rg}}, \mathrm{S}_{\mathrm{Dg}}$ e $\mathrm{S}_{\mathrm{pH}}$ - Índices de susceptibilidade do comprimento radicular, da altura do dossel, da matéria seca das raízes e do dossel e da alteração do pH da solução nutritiva, respectivamente, em função da susceptibilidade das linhagens de arroz de terras altas à toxicidade do alumínio

1 Médias seguidas pela mesma letra, na coluna, não diferiram significativamente, a nível de $5 \%$ de probabilidade, pelo teste de Scott-Knott 
Tabela 2. Coeficiente de correlação entre os indicadores de susceptibilidade do arroz de terras altas à toxicidade de alumínio, massa da matéria seca das raízes (Raízes $s_{\mathrm{g}}$ ) e do dossel (Plantas $s_{\mathrm{g}}$ ), comprimento das raízes (Raízes $\mathrm{cm}_{\mathrm{cm}}$ ) e do dossel (Plantas ${ }_{\mathrm{cm}}$ ) das linhagens de arroz de terras altas, $\mathrm{pH}$ final da solução nutritiva $\left(\mathrm{pH}_{\text {final }}\right)$, em $40 \mathrm{mg} \mathrm{dm}$-3 de alumínio e índices de susceptibilidade ao alumínio*

\begin{tabular}{|c|c|c|c|c|c|c|c|c|c|}
\hline Indicador & Ráizes $_{g}$ & Plantas $_{g}$ & Ráizes $_{\mathrm{cm}}$ & Plantas $_{c m}$ & $\mathrm{PH}_{\text {ind }}$ & $\mathrm{S}_{\mathrm{Rcm}}$ & $S_{D c m}$ & $\mathrm{~S}_{\mathrm{Rg}}$ & $S_{D g}$ \\
\hline Plantas $_{g}$ & $\begin{array}{r}0,6282 \\
<0,0001\end{array}$ & & & & & & & & \\
\hline Ráizes $_{\mathrm{cm}}$ & $\begin{array}{l}0,2284 \\
0,0008\end{array}$ & $\begin{array}{l}0,1417 \\
0,0392\end{array}$ & & & & & & & \\
\hline Plantas $_{\mathrm{cm}}$ & $\begin{array}{l}0,2243 \\
0,0010\end{array}$ & $\begin{array}{l}0,2180 \\
0,0014\end{array}$ & $\begin{array}{r}0,4115 \\
<0,0001\end{array}$ & & & & & & \\
\hline $\mathrm{pH}_{\text {ind }}$ & $\begin{array}{l}0,0095 \\
0,8901\end{array}$ & $\begin{array}{r}-0,0518 \\
0,4529\end{array}$ & $\begin{array}{r}-0,0232 \\
0,7365\end{array}$ & $\begin{array}{r}-0,0538 \\
0,4360\end{array}$ & & & & & \\
\hline $\mathrm{S}_{\mathrm{Rcm}}$ & $\begin{array}{r}-0,2255 \\
0,0009\end{array}$ & $\begin{array}{r}-0,1526 \\
0,0262\end{array}$ & $\begin{array}{r}-0,8282 \\
<0,0001\end{array}$ & $\begin{array}{r}-0,3629 \\
<0,0001\end{array}$ & $\begin{array}{r}-0,0336 \\
0,6066\end{array}$ & & & & \\
\hline $\mathrm{S}_{\mathrm{Dcm}}$ & $\begin{array}{r}-0,1670 \\
0,0149\end{array}$ & $\begin{array}{r}-0,1211 \\
0,0785\end{array}$ & $\begin{array}{r}-0,4340 \\
<0,0001\end{array}$ & $\begin{array}{r}-0,8732 \\
<0,0001\end{array}$ & $\begin{array}{l}0,0958 \\
0,1644\end{array}$ & $\begin{array}{r}0,4012 \\
<0,0001\end{array}$ & & & \\
\hline $\mathrm{S}_{\mathrm{Rg}}$ & $\begin{array}{r}-0,8419 \\
<0,0001\end{array}$ & $\begin{array}{r}-0,5538 \\
<0,0001\end{array}$ & $\begin{array}{r}-0,0233 \\
0,0006\end{array}$ & $\begin{array}{r}-0,2558 \\
0,0002\end{array}$ & $\begin{array}{r}-0,0046 \\
0,9470\end{array}$ & $\begin{array}{r}0,3535 \\
<0,0001\end{array}$ & $\begin{array}{r}0,2347 \\
<0,0006\end{array}$ & & \\
\hline $\mathrm{S}_{\mathrm{Dg}}$ & $\begin{array}{r}-0,6215 \\
<0,0001\end{array}$ & $\begin{array}{r}-0,7856 \\
<0,0001\end{array}$ & $\begin{array}{r}-0,2086 \\
0,0023\end{array}$ & $\begin{array}{r}-0,2429 \\
0,0004\end{array}$ & $\begin{array}{l}0,0807 \\
0,2422\end{array}$ & $\begin{array}{r}0,3471 \\
<0,0001\end{array}$ & $\begin{array}{r}0,2445 \\
<0,0003\end{array}$ & $\begin{array}{r}0,7967 \\
<0,0001\end{array}$ & \\
\hline $\mathrm{S}_{\mathrm{pH}}$ & $\begin{array}{r}0,3427 \\
<0,0001\end{array}$ & $\begin{array}{l}0,2018 \\
0,0032\end{array}$ & $\begin{array}{l}0,2280 \\
0,0008\end{array}$ & $\begin{array}{r}0,2647 \\
<0,0001\end{array}$ & $\begin{array}{r}-0,3328 \\
<0,0001\end{array}$ & $\begin{array}{r}-0,2159 \\
<0,0016\end{array}$ & $\begin{array}{l}-0,3072 \\
<0,0001\end{array}$ & $\begin{array}{r}-0,3967 \\
<0,0001\end{array}$ & $\begin{array}{r}-0,2796 \\
<0,0001\end{array}$ \\
\hline
\end{tabular}

${ }^{*} \mathrm{~S}_{\mathrm{Rcm}}, \mathrm{S}_{\mathrm{Dcm}}, \mathrm{S}_{\mathrm{Rg}}, \mathrm{S}_{\mathrm{Dg}}$ e $\mathrm{S}_{\mathrm{pH}}$ - Índices de susceptibilidade do comprimento radicular, da altura do dossel, da matéria seca das raízes e do dossel e da alteração do $\mathrm{pH}$ da solução nutritiva, respectivamente, em função da susceptibilidade das linhagens de arroz de terras altas à toxicidade do alumínio. Os valores na segunda linha apresentam os a probabilidade da significância do coeficiente de correlação.

\section{CONCLUSÕES}

1. O índice de susceptibilidade do crescimento radicular à toxicidade de alumínio mostrou-se eficiente na avaliação da resistência diferenciada das linhagens à toxicidade de alumínio.

2. As linhagens CNA4120, CNA4164 e CNA1383 apresentam raízes bem desenvolvidas, tanto na ausência como na presença de alumínio, enquanto as linhagens CNA 6430, CNA4108, CNA4166, CNA4181, CNA5164, CNA5165, CNA4105, CNA1168, CNA4125 e CNA4150 apontam raízes bem desenvolvidas na ausência de toxicidade de alumínio; entretanto, não suportam a presença tóxica do mesmo.

\section{AGRADECIMENTOS}

Aos estagiários Fernando Xavier da Silva e Kin Carlos Gomides, pelo auxílio na condução desta pesquisa.

\section{LITERATURA CITADA}

Bennet, R.J.; Breen, C.M.; Fey, M.V. Aluminum uptake sites in the primary root of Zea mays L. South African Journal of Plant and Soil, Pretoria, v.2, n.1, p.1-7, 1985.

Clarkson, D.T. The effect of aluminum and some other trivalent metal cations on cell division in the root apices of Allium cepa. Annals of Botany, London, v.29, n.114, p.309$315,1965$.
Delhaize E.; Ryan P.R.; Randall P.J. Aluminum tolerance in wheat (Triticum aestivum L.). II. Aluminum-stimulated excretion of malic acid from root apices. Plant Physiology, Bethesda, v.103, n.3, p.695-702, 1993.

Fageria, N.K. Tolerância diferencial de cultivares de arroz ao alumínio em solução nutritiva. Pesquisa Agropecuária Brasileira, Brasília, v.17, n.1, p.1-9, 1982.

Fageria, N.K. Influence of aluminum in nutrient solutions on chemical composition of two rice cultivars at different growth stages. Plant and Soil, The Hague, v.85, n.3, p.423-429, 1985.

Fageria, N.K.; Baligar, V.C.; Writh, R.J. Aluminum toxicity in crop plants. Journal Plant Nutrition, New York, v.11, n.3, p.303-319, 1988.

Fageria, N.K.; Zimmermann, F.J.P. Seleção de cultivares de arroz para tolerância a toxidez de alumínio em solução nutritiva. Pesquisa Agropecuária Brasileira, Brasília, v.14, n.2, p.141-147, 1979.

Fisher, R.A.; Maurer, R. Drought resistance in spring wheat cultivars. I. Grain yield responses. Australian Journal of Agricultural Research, Victoria, v.29, n.5, p.897-912, 1978.

Foy, C.D. Plant adaptation to acid, aluminum-toxic soils. Communications in Soil Science and Plant Analysis, New York, v.19, n.7-12, p.959-987, 1988.

Foy, C.D. Soil chemical factors limiting plant root growth. In: Hatfield, J.L.; Stewart, B.A. (eds.). Limitations to plant root growth. New York: Springer, 1992. p.97-149.

R. Bras. Eng. Agríc. Ambiental, v.10, n.4, p.855-860, 2006. 
Foy, C.D.; Burns, G.R.; Brown J.C.; Fleming, A.L. Differential aluminum tolerance of two wheat varieties associated with plant-induced $\mathrm{pH}$ changes around their roots, Soil Science Society of America Proceedings, Madison, v.29, n.1, p.64-67, 1965.

Furlani, A.M.C.; Furlani, P.R. Composição e pH de soluções nutritivas para estudos fisiológicos e seleção de plantas em condições nutricionais adversas. Campinas: Instituto Agronômico, 1988. 34p. Boletim Técnico, 121

Horst, W.J.; Wagner, A.; Marschner, H. Mucilage protects root meristems from aluminum injury. Zeitschrift Fuer Pflanzenphysiologie, Stuttgart, v.105, p.435-444, 1982.

Kochian, L.V. Cellular mechanisms of aluminum toxicity and resistance in plants. Annual Review of Plant Physiology and Plant Molecular Biology, Palo Alto, v.46, p.237-260, 1995.

Li, X.F.; Ma J.F.; Hiradate S.; Matsumoto, H. Mucilage strongly binds aluminum but does not prevent roots from aluminum injury in Zea mays. Physiologia Plantarum, Copenhagen, v.108, n.2, p.152-160, 2000.

Mendonça, R.J.; Cambraia, J.; Oliva, M.A.; Oliveira, J.A. de. Capacidade de cultivares de arroz de modificar o $\mathrm{pH}$ de soluções nutritivas na presença de alumínio. Pesquisa Agropecuária Brasileira, Brasília, v.40, n.5, p.447-452, 2005.
Mendonça, R.J.; Cambraia, J.; Oliveira, J.A. de; Oliva, M.A. Efeito do alumínio na absorção e na utilização de macronutrientes em duas cultivares de arroz. Pesquisa Agropecuária Brasileira, Brasília, v.38, n.7, p.843-848, 2003.

Miyasake, S.C.; Hawes, M.C. Possible role of root border ce1ls in detection and avoidance of aluminum toxicity. Plant Physiology, Bethesda, v.125, n.4, p.1978-1987, 2001.

Pellet, D.M.; Grunes, D.L.; Kochian, L.V. Organic acid exudation as an aluminum-tolerance mechanism in maize (Zea mays L.). Planta: An International Journal of Plant Biology, New York, v.196, n.4, p.788-795, 1995.

Rout, G. R.; Samantaray, S.; Das, P. Aluminum toxicity in plants: a review. Agronomie, Paris, v.21, n.1, p.3-21, 2001.

Sivaguru, M.; Paliwal, K. Differential aluminum tolerance in some tropical rice cultivars. II. Mechanism of aluminum tolerance. Journal of Plant Nutrition, New York, v.16, n.9, p.1717-1732, 1993.

Taylor, G.J. Current views of the aluminum stress response: the physiological basis of tolerance. In: Randall, D.D.; Blevins, D.G.; Miles, C.D. (ed.). Current topics in plant biochemistry and physiology. Columbia: University of Missouri, 1991. v.10, p.57-93. 